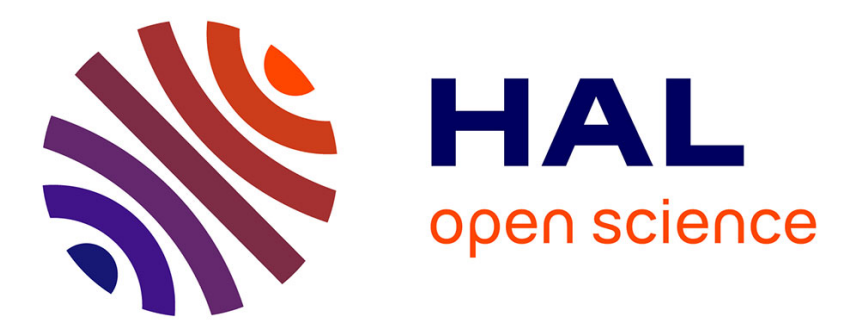

\title{
Eddy-current testing with the Expected Improvement optimization algorithm
}

Sandor Bilicz, Emmanuel Vazquez, Jozsef Pavo, Marc Lambert, Szabolcs

\author{
Gyimothy
}

\section{To cite this version:}

Sandor Bilicz, Emmanuel Vazquez, Jozsef Pavo, Marc Lambert, Szabolcs Gyimothy. Eddy-current testing with the Expected Improvement optimization algorithm. 15th IFAC Symposium on System Identification, SYSID 2009, Jul 2009, Saint-Malo, France. hal-00446202

\section{HAL Id: hal-00446202 https://hal.science/hal-00446202}

Submitted on 12 Jan 2010

HAL is a multi-disciplinary open access archive for the deposit and dissemination of scientific research documents, whether they are published or not. The documents may come from teaching and research institutions in France or abroad, or from public or private research centers.
L'archive ouverte pluridisciplinaire HAL, est destinée au dépôt et à la diffusion de documents scientifiques de niveau recherche, publiés ou non, émanant des établissements d'enseignement et de recherche français ou étrangers, des laboratoires publics ou privés. 


\title{
Eddy-current testing with the Expected Improvement optimization algorithm
}

\author{
Sándor Bilicz ${ }^{*, * *}$ Emmanuel Vazquez ${ }^{* *}$ József Pávó* Marc Lambert*** \\ Szabolcs Gyimóthy* \\ * Budapest University of Technology and Economics, Egry J. u. 18, H-1521 \\ Budapest, Hungary(e-mail: biliczs@sch.bme.hu) \\ ** SUPELEC, 91192 Gif-sur-Yvette cedex, France \\ *** Département de Recherche en Électromagnétisme, Laboratoire des \\ Signaux et Systèmes UMR8506 (CNRS-SUPELEC-Univ Paris Sud 11), 91192 \\ Gif-sur-Yvette cedex, France
}

\begin{abstract}
This paper presents an inverse problem methodology in the domain of non-destructive testing, and more precisely eddy-current testing. Our objective is to use a precise but expensive-to-evaluate model of the electromagnetic induction phenomenon in a conductive material and to estimate the characteristics of a flaw by minimization of a regularized criterion with the Expected Improvement (EI) global optimization algorithm. The EI algorithm is designed to estimate a global optimum of a function with a restricted budget of function evaluations. Thus, we expect to be able to estimate the characteristics of a flaw with a relatively low cost despite resorting to an expensive model of the induction phenomenon. The efficiency of the approach is discussed in the light of preliminary numerical examples obtained using synthetic data.
\end{abstract}

Keywords: eddy current testing; inversion; global optimization; Gaussian processes; Expected Improvement algorithm

\section{INTRODUCTION}

This paper presents an inverse problem methodology in the domain of non-destructive testing, and more precisely eddycurrent testing (ECT). As illustrated by a large number of contributions in the last ten years, the estimation of the characteristics of scattering objects remains quite challenging from a theoretical, computational and/or experimental perspective, even in the case of the simplest configurations (e.g., a homogeneous embedding space). In this paper, our objective is to use a precise but expensive-to-evaluate model of the electromagnetic induction phenomenon in a conductive material. Then, to estimate the characteristics of a flaw, a regularized criterion is minimized using the Expected Improvement (EI) global optimization algorithm. The EI algorithm is designed to estimate a global optimum of a function with a restricted budget of function evaluations. Thus, we expect to be able to estimate the characteristics a flaw with a relatively low cost despite resorting to an expensive model of the induction phenomenon.

The EI algorithm is well-known in the domain of global optimization, but to the best of our knowledge, using the EI algorithm for the ECT problem remains largely unexplored. Preliminary results for the characterization of a single volumetric defect have been presented by the authors in [Bilicz et al., 2008]. In this paper, a more challenging case is studied. We consider two infinitesimally thin material defects close to each other. Our contribution is twofold. First, from the point of view of ECT, we would like to show that the methodology we propose is promising. More generally, from the point of view of the inverse problem community, we would like to bring attention to an efficient optimization algorithm that renders practical the use of precise but expensive direct models.
The paper is organized as follows. First, we present the theoretical formulation of the direct problem and some aspects of its numerical implementation ( $(2)$. Then the inverse problem - along with our regularizing assumptions - will be presented (§ 3). Next the new stochastic approach and the optimization task will be in the focus ( $\$ 4)$. Finally, numerical examples will illustrate the proposed method and the conclusions of the experiments will be drawn $(\S 5)$.

\section{THE FORWARD PROBLEM}

Assume the arrangement shown in Fig. 1 where two infinitesimally thin cracks located in the planar surfaces $S_{1}$ and $S_{2}$ inside a non-ferromagnetic plate are searched by a pancake type ECT coil. The normal vectors of the $S_{1}$ and $S_{2}$ surfaces are $\hat{\boldsymbol{n}}_{1}$ and $\hat{\boldsymbol{n}}_{2}$, respectively. In this Subsection the method used for the solution of the forward problem is discussed. In other words, the method used for the calculation of the change of the impedance of the ECT coil due to the presence of the cracks $(\Delta Z)$ is described when the location and the orientation of $S_{1}$ and $S_{2}$ are arbitrary. During the solution of the inverse problem, however it will be assumed that the cracks are outer defects (OD) and they are parallel to each other, consequently $\hat{\boldsymbol{n}}_{1}=\hat{\boldsymbol{n}}_{2}=\hat{\boldsymbol{x}}$ ( $\hat{\boldsymbol{x}}$ is the unit vector of the $x$ co-ordinate axis). Fig. 1 shows the particular arrangement that is actually assumed for the inverse problem.

The electromagnetic interaction of the exciting coil with nonconducting cracks whose thickness are considerably smaller than their other dimensions might be calculated based on the so-called infinitesimally thin crack approximation described by Bowler [1994]. This model assumes the cracks in the otherwise homogeneous conductor as mathematical surfaces that block the flow of the eddy currents across their surfaces, in other 

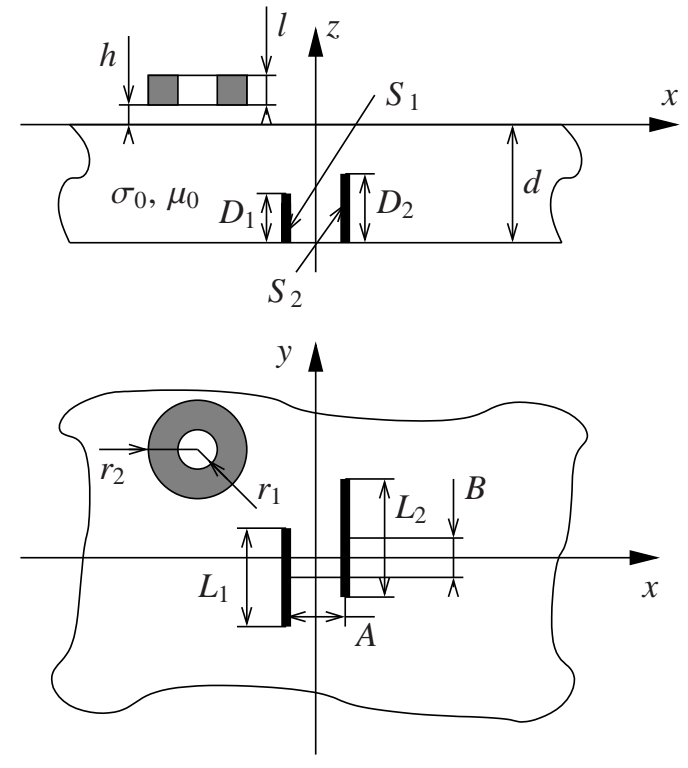

Fig. 1. Schematic drawing of the geometry of the investigated forward and inverse problems

words the normal component of the electric field must be zero on the surfaces representing the cracks. In the same time, the magnetic field is assumed to be continuous on the two sides of these surfaces.

The above boundary conditions might be satisfied by replacing the $S_{1}$ and $S_{2}$ mathematical surfaces with current dipole distributions, $p_{1}(\boldsymbol{r}) \hat{\boldsymbol{n}}_{1}$ and $p_{2}(\boldsymbol{r}) \hat{\boldsymbol{n}}_{2}$, having the same effect as the presence of the crack (the function $p_{1}(\boldsymbol{r})$ and $p_{2}(\boldsymbol{r})$ are non-zero only on the surfaces $S_{1}$ and $S_{2}$, respectively). Thus, the sum of the electromagnetic field generated by the exciting coil and the secondary sources in the homogeneous conductor will provide the field generated by the crack-probe interaction. Consequently this field must satisfy the above boundary conditions. In the case of cracks with planar surfaces, the described consideration leads to the following integral equation reported by Pávó [2000]:

$$
\begin{aligned}
0=E_{n 1}^{i}\left(\boldsymbol{r}_{1}\right)-j \omega \mu_{0} \iint_{S_{2}} g_{21}\left(\boldsymbol{r}_{1} \mid \boldsymbol{r}^{\prime}\right) p_{2}\left(\boldsymbol{r}^{\prime}\right) d \boldsymbol{r}^{\prime}- \\
\quad-j \omega \mu_{0} \lim _{\boldsymbol{r} \rightarrow \boldsymbol{r}_{1 \pm}} \iint_{S_{1}} g_{11}\left(\boldsymbol{r} \mid \boldsymbol{r}^{\prime}\right) p_{1}\left(\boldsymbol{r}^{\prime}\right) d \boldsymbol{r}^{\prime}, \quad \boldsymbol{r}_{1} \in S_{1}, \\
0=E_{n 2}^{i}\left(\boldsymbol{r}_{2}\right)-j \omega \mu_{0} \iint_{S_{1}} g_{12}\left(\boldsymbol{r}_{2} \mid \boldsymbol{r}^{\prime}\right) p_{1}\left(\boldsymbol{r}^{\prime}\right) d \boldsymbol{r}^{\prime}- \\
\quad-j \omega \mu_{0} \lim _{\boldsymbol{r} \rightarrow \boldsymbol{r}_{2 \pm}} \iint_{S_{2}} g_{22}\left(\boldsymbol{r} \mid \boldsymbol{r}^{\prime}\right) p_{2}\left(\boldsymbol{r}^{\prime}\right) d \boldsymbol{r}^{\prime}, \quad \boldsymbol{r}_{2} \in S_{2},
\end{aligned}
$$

where $\boldsymbol{r}_{k \pm}(k=1,2)$ denotes the limiting values of the approaches towards the point $\boldsymbol{r}_{k}$ from the $\hat{\boldsymbol{n}}_{k}$ or $-\hat{\boldsymbol{n}}_{k}$ directions, $\omega$ is the angular frequency of the sinusoidal excitation of the probe coil and $\mu_{0}$ is the permeability of the vacuum. $E_{n k}^{i}=\hat{\boldsymbol{n}}_{k} \cdot \boldsymbol{E}^{i}$ $(k=1,2)$, where $\boldsymbol{E}^{i}$ is the so-called incident field that is the electric field generated by the current of the exciting coil in the defect-free plate. $g_{k l}\left(\boldsymbol{r} \mid \boldsymbol{r}^{\prime}\right)=\hat{\boldsymbol{n}}_{l} \cdot \mathbf{G}\left(\boldsymbol{r} \mid \boldsymbol{r}^{\prime}\right) \cdot \hat{\boldsymbol{n}}_{k}(k, l=1,2)$ where $\mathbf{G}\left(\boldsymbol{r} \mid \boldsymbol{r}^{\prime}\right)$ is the Green's dyad transforming the current excitation into the electric field.

The surfaces of the cracks are discretized on a regular lattice where the unknown dipole density function is approximated by a piecewise linear function. The integral equation is tested by first order testing functions and the kernel of the integral equation is evaluated by the formulae published by Pávó and Miya [1994]. After solving the linear system of equations obtained by the described discretization of the integral equations (1)-(2), the dipole density function can be calculated. Knowing $p_{1}(\boldsymbol{r})$ and $p_{2}(\boldsymbol{r})$, the impedance variation of the probe, $\Delta Z$, due to the presence of the cracks can be calculated based on the reciprocity theorem [Bowler, 1994]:

$$
\Delta Z=-\frac{1}{I^{2}} \sum_{k=1}^{2} \iint_{S_{k}} E_{n k}^{i}(\boldsymbol{r}) p_{k}(\boldsymbol{r}) d \boldsymbol{r},
$$

where $I$ is the current of the exciting coil.

The results of the described solution of the forward problem are tested against measurements available for single artificial cracks (unfortunately measured results on two parallel cracks are not available in the literature). The comparison verified the validity of the results.

For rectangular shaped cracks being parallel to each other and being perpendicular to the surface of the plate, the integral equations (1)-(2) can be solved by approximating $p_{1}(\boldsymbol{r})$ and $p_{2}(\boldsymbol{r})$ with the function series introduced in [Pávó and Lesselier, 2006]. Using this method, the results on several test configurations are very close to those obtained by the method described above. The good agreement supports the validity of both solution methods.

\section{THE INVERSE PROBLEM}

When the probe coil scans the zone containing the cracks, the variations of the coil impedance are measured at each point of a regular rectangular grid, above the supposedly damaged zone of the plate. In other words, a so-called surface scan is performed. The scan consists of $N$ coil locations where the measured impedance variations are $\Delta \mathcal{Z}_{k}, k=1,2, \ldots, N$.

The problem is to characterize the flaw from this set of measured impedance variations, which will be viewed as the input data of the inverse problem.

\subsection{Regularizing assumptions}

It is customary to introduce some regularizing assumptions to reduce the ill-posedness of the inverse problem. In this work, two rectangular shaped thin cracks are assumed in the plate specimen, under (or near) the surface scan measurement (see Fig. 1). Both cracks are of OD type. Their orientation is supposedly known, together with the "geometrical center" of the two-cracks-system (i.e. the distances of the crack midpoints from the origin are the same for both cracks in the $x(A / 2)$ and in the $y(B / 2)$ directions as well). As a result, only 6 geometrical parameters are needed to describe the configuration: the length of the cracks $\left(L_{1}, L_{2}\right)$, their depth $\left(D_{1}, D_{2}\right)$ and the $x-y$ distances of their centers $(A, B)$, respectively (see Fig. 1). To simplify the notations, the unknown defect parameters are collected in a vector $\mathbf{t}$ :

$$
\mathbf{t}=\left[A, B, L_{1}, L_{2}, D_{1}, D_{2}\right] .
$$

The definition domain of $\mathbf{t}$ will be denoted by $\mathbb{T}$, and will be referred to as the parameter space.

The forward solver ( $\S 2$ ) is used to compute the impedance variation due to a fault characterized by its parameter vector $\mathbf{t}$. A simulated surface scan consists of such computed impedance variations, denoted as $\Delta Z_{k}(\mathbf{t}), k=1,2, \ldots, N$. Obviously, the 
simulation is performed at exactly the same coil positions as the real measurement, thus both the real and the simulated surface scan consists of $N$ coil locations.

\subsection{Optimization task}

The next step for solving the inverse problem is to achieve the strongest resemblance between the impedance signal obtained by simulation, $\left\{\Delta Z_{k}(\mathbf{t}), k=1, \ldots, N\right\}$, and the measurement, $\left\{\Delta \mathcal{Z}_{k}, k=1, \ldots, N\right\}$, by tuning the parameter vector $\mathbf{t}$.

The applied inversion method requires a finite parameter space $\mathbb{T}$, meaning that one has to set lower and upper bounds for each parameter and, moreover, the parameter axes are needed to be discretized. Consequently, the space $\mathbb{T}$ has to consist of a finite number of parameter points $\mathbf{t}$. The bounds and discretization of each parameter are summarized in Table 1. The parameters are independent from each other, thus, the parameter space $\mathbb{T}$ can be imagined as a 6 dimensional hypercube, spanned by the 6 parameter axes. The hypercube includes $5^{2} \cdot 7^{4}=60025$ parameter points.

Table 1. Discretization of the parameter axes.

\begin{tabular}{|l||c|c|c|c|c|c|}
\hline Parameter & $A$ & $B$ & $L_{1}$ & $L_{2}$ & $D_{1}$ & $D_{2}$ \\
\hline \hline Minimum value $(\mathrm{mm})$ & 0.25 & 0 & 2 & 2 & 0.1250 & 0.1250 \\
\hline Maximum value $(\mathrm{mm})$ & 1.25 & 4 & 8 & 8 & 0.8750 & 0.8750 \\
\hline Step size $(\mathrm{mm})$ & 0.25 & 1 & 1 & 1 & 0.0125 & 0.0125 \\
\hline No. of points & $\mathbf{5}$ & $\mathbf{5}$ & $\mathbf{7}$ & $\mathbf{7}$ & $\mathbf{7}$ & $\mathbf{7}$ \\
\hline
\end{tabular}

To give a mathematical form to the resemblance between two surface-scan impedance signals, we define the similarity function

$$
Q(\mathbf{t})=\frac{\sum_{k=1}^{N}\left|\Delta Z_{k}(\mathbf{t})-\Delta \mathcal{Z}_{k}\right|^{2}}{\sum_{k=1}^{N}\left|\Delta \mathcal{Z}_{k}\right|^{2}} .
$$

Our objective is thus to minimize $Q(\mathbf{t})$, i.e. to find

$$
\hat{\mathbf{t}}=\arg \min _{\mathbf{t} \in \mathbb{T}} Q(\mathbf{t}) \text {. }
$$

\section{STOCHASTIC MODELING AND OPTIMIZATION OF THE SIMILARITY FUNCTION}

Our objective is to implement the Expected Improvement algorithm to minimize $Q(\mathbf{t})$. One iteration of such an algorithm involves mainly two steps:

(1) the construction of an approximation of the similarity function from a set of past evaluations of the function obtained at previous iterations. To this end, a random process is chosen as a model of the similarity function and an interpolation by kriging is performed.

(2) the search of the maximum of the Expected Improvement over the parameter space $\mathbb{T}$ then the computation of the similarity function at that parameter point.

This two-step procedure is repeated iteratively until a stopping criterion is met, i.e. a sequential optimization algorithm is obtained.

\subsection{Kriging interpolation}

Let us assume that we have already observed the multivariate scalar function $Q(\mathbf{t})$ at $n$ points $\mathbf{t}_{1}, \mathbf{t}_{2}, \ldots, \mathbf{t}_{n}$ of $\mathbb{T}$. In this way, $n$ function values $Q_{1}=Q\left(\mathbf{t}_{1}\right), Q_{2}=Q\left(\mathbf{t}_{2}\right), \ldots, Q_{n}=Q\left(\mathbf{t}_{n}\right)$ are known. We would like to predict the function value at unobserved sites. One method to achieve this goal is to use kriging, a random process approach developed in the 60s in geostatistics [Chiles and Delfiner, 1999]. The method is also well-known for modeling computer simulations [Sacks et al., 1989].

Let $\xi(\mathbf{t})$ be a Gaussian random process that models the function $Q(\mathbf{t})$. Thus, each observation $Q_{k}$ is considered as the realization of the Gaussian random variable $\xi\left(\mathbf{t}_{k}\right)(k=1,2, \ldots, n)$. Kriging computes the best linear unbiased predictor $(B L U P)$ of $\xi(\mathbf{t})$. Let us denote this prediction by $\hat{\xi}(\mathbf{t})$. The predictor is linear in the sense that it is a linear combination of the observed random variables $\xi\left(\mathbf{t}_{k}\right), k=1,2, \ldots, n$, which can therefore be written as

$$
\hat{\xi}(\mathbf{t})=\sum_{k=1}^{n} \lambda_{k}(\mathbf{t}) \xi\left(\mathbf{t}_{k}\right) .
$$

Unbiasedness relates to the fact that the mean of $\hat{\xi}(\mathbf{t})$ is equal to the mean of $\xi(\mathbf{t})$, i.e. the mean prediction error is zero:

$$
\mathbb{E}[\varepsilon(\mathbf{t})]=\mathbb{E}[\hat{\xi}(\mathbf{t})-\xi(\mathbf{t})]=0 .
$$

The term "best" means that the prediction error $\varepsilon(\mathbf{t})$ of the kriging predictor has the smallest variance among all unbiased predictors. This variance (also called kriging error) may be written as

$$
\hat{\sigma}^{2}(\mathbf{t})=\operatorname{var}[\hat{\xi}(\mathbf{t})-\xi(\mathbf{t})]=\mathbb{E}\left[(\hat{\xi}(\mathbf{t})-\xi(\mathbf{t}))^{2}\right],
$$

using the unbiasedness condition (6).

The objective is to find the coefficients $\lambda_{k}(\mathbf{t})$ in (5) that provide the BLUP. The kriging error can be written using the covariance function, which describes the dependence between two random variables of the process at different points. Let us denote the covariance function by $k\left(\mathbf{t}_{a}, \mathbf{t}_{b}\right)=\operatorname{cov}\left[\xi\left(\mathbf{t}_{a}\right), \xi\left(\mathbf{t}_{b}\right)\right]$, where $\mathbf{t}_{a}$ and $\mathbf{t}_{b}$ are two points in $\mathbb{T}$. Let us denote by $\mathbf{K}$, the covariance matrix whose entries correspond to the covariances of the random process between the observation points $\mathbf{t}_{1}, \mathbf{t}_{2}, \ldots, \mathbf{t}_{n}$ :

$$
\mathbf{K}=\left[\begin{array}{cccc}
k\left(\mathbf{t}_{1}, \mathbf{t}_{1}\right) & k\left(\mathbf{t}_{1}, \mathbf{t}_{2}\right) & \ldots & k\left(\mathbf{t}_{1}, \mathbf{t}_{n}\right) \\
k\left(\mathbf{t}_{2}, \mathbf{t}_{1}\right) & k\left(\mathbf{t}_{2}, \mathbf{t}_{2}\right) & \ldots & k\left(\mathbf{t}_{2}, \mathbf{t}_{n}\right) \\
\vdots & \vdots & \ddots & \vdots \\
k\left(\mathbf{t}_{n}, \mathbf{t}_{1}\right) & k\left(\mathbf{t}_{n}, \mathbf{t}_{2}\right) & \ldots & k\left(\mathbf{t}_{n}, \mathbf{t}_{n}\right)
\end{array}\right]
$$

If one has some prior knowledge on the function to be modeled it can be reflected by giving a prior mean to the predictor. Since in our case no information is available but the observed function values, a constant (but unknown) mean $\mathbb{E}[\xi(\mathbf{t})]=C$ is assumed.

To simplify the notations let us collect the coefficients $\lambda_{k}(\mathbf{t})$ into a vector $\lambda(\mathbf{t})=\left[\begin{array}{llll}\lambda_{1}(\mathbf{t}) & \lambda_{2}(\mathbf{t}) & \ldots & \lambda_{n}(\mathbf{t})\end{array}\right]^{\top}$, and denote by $\mathbf{k}(\mathbf{t})$ the vector whose elements are the values of the covariance between $\mathbf{t}$ and the observation points: $\mathbf{k}(\mathbf{t})=$ $\left[k\left(\mathbf{t}, \mathbf{t}_{1}\right) k\left(\mathbf{t}, \mathbf{t}_{2}\right) \ldots k\left(\mathbf{t}, \mathbf{t}_{n}\right)\right]^{\top}$. It can be shown that the determination of $\boldsymbol{\lambda}(\mathbf{t})$ boils down to computing the solution of the linear system of equations (see, e.g., Villemonteix et al. [2008]):

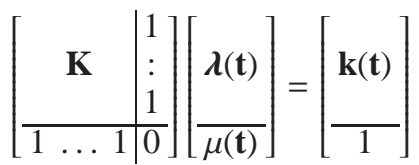

where $\mu(\mathbf{t})$ is the Lagrange multiplier, which corresponds to the enforcement of the unbiasedness condition.

Once the vector $\lambda(\mathbf{t})$ has been computed, a predicted value of the similarity function $Q(\mathbf{t})$ can be written as 


$$
\hat{Q}(\mathbf{t})=\sum_{k=1}^{n} \lambda_{k}(\mathbf{t}) Q_{k}
$$

It is easy to show that the function $\mathbf{t} \mapsto \hat{Q}(\mathbf{t})$ interpolates $Q(\mathbf{t})$ at observed points. An interesting property of kriging, is that an estimate of the uncertainty of the prediction is available via the kriging error, which can be written as

$$
\hat{\sigma}^{2}(\mathbf{t})=k(\mathbf{t}, \mathbf{t})-\boldsymbol{\lambda}(\mathbf{t})^{\top} \mathbf{k}(\mathbf{t})-\mu(\mathbf{t}) .
$$

This feature will be essential in the adaptive sampling strategy of the Expected Improvement algorithm.

\subsection{Covariance model}

Before focusing on the optimization algorithm, we mention very briefly how the covariance function is chosen in practice (see also Villemonteix et al. [2008]).

First, a simplifying assumption is made - as usually done in geostatistics (see, e.g., Chiles and Delfiner [1999]) - namely that the random process is stationary. Then, the covariance function is a univariate function $k(h)$ where $h$ is a distance between two points $\mathbf{t}_{a}, \mathbf{t}_{b} \in \mathbb{T}$. This distance needs not to be the classical Euclidean distance. In our case, the components of $\mathbf{t} \in \mathbb{T}$ are of different kinds. Thus, it is reasonable to use some anisotropic distance, which may be written as

$$
h=\sqrt{\sum_{d=1}^{D}\left(\frac{t_{a, d}-t_{b, d}}{\rho_{d}}\right)^{2}} .
$$

where $t_{a, d}$ and $t_{b, d}$ are the $d^{\text {th }}$ components of the vectors $\mathbf{t}_{a}$ and $\mathbf{t}_{b}$, respectively, and $D$ the dimension of $\mathbb{T}$. The parameters $\rho_{d}, d=1,2, \ldots, D$, represent the range of the covariance, or the typical correlation distance, in the direction of the $d^{\text {th }}$ component.

Second, a parameterized covariance function is chosen and its parameters are estimated using the data with a maximum likelihood method (see, e.g, Villemonteix et al. [2008]). We use the Matérn covariance function, which can be written as

$$
k(h)=\frac{\sigma^{2}}{2^{v-1} \Gamma(v)}(2 \sqrt{v} h)^{v} \mathcal{K}_{v}(2 \sqrt{v} h),
$$

where $\mathcal{K}_{v}$ is the modified Bessel function of the second kind of order $v$. The parameter $v$ controls the regularity of the random process - the higher the $v$, the more regular the process is. The parameter $\sigma^{2}$ is the variance of the process $\left(k(0)=\sigma^{2}\right)$. The parameters $\rho_{d}$ in (12) are also estimated by maximum likelihood (the covariance function has indeed $D+2$ parameters).

\subsection{Expected Improvement}

Since the similarity function $Q$ (to be minimized) requires to compute the solution of a forward problem, the computational cost of evaluating $Q$ is non-negligible. Moreover, for an inversion method to be useful in practice, the computational burden of the method must not be too high. Thus, we wish to limit the number of evaluations of $Q$ in the inversion procedure, which means that the optimization method used to minimize $Q$ must be efficient.

The expected improvement (EI) algorithm is an iterative method to find the global minimizers of an expensive-to-evaluate function [Jones, 2001]. The method is based on interpolation by kriging of the function to be optimized. Let us assume that
Table 2. Parameters of the ECT configuration.

\begin{tabular}{|l|l|l|l|}
\hline \multicolumn{5}{|c|}{ Metal plate } \\
\hline Thickness $(d)$ & $1.25 \mathrm{~mm}$ & Conductivity $\left(\sigma_{0}\right)$ & $10^{6} \mathrm{~S} / \mathrm{m}$ \\
\hline \multicolumn{4}{|c|}{ Probe coil } \\
\hline Inner radius $\left(r_{1}\right)$ & $0.6 \mathrm{~mm}$ & Outer radius $\left(r_{2}\right)$ & $1.6 \mathrm{~mm}$ \\
Height $(l)$ & $0.8 \mathrm{~mm}$ & Lift-off $(h)$ & $0.5 \mathrm{~mm}$ \\
No. of turns & 140 & Frequency & $150 \mathrm{kHz}$ \\
\hline \multicolumn{4}{|c|}{ Surface scan } \\
\hline Points in the $x$ dir. & 11 & Points in the y dir. & 41 \\
Step in the $x$ dir. & $0.5 \mathrm{~mm}$ & Step in the y dir. & $0.5 \mathrm{~mm}$ \\
\hline
\end{tabular}

$Q$ has been evaluated at $n$ points $Q_{1}=Q\left(\mathbf{t}_{1}\right), Q_{2}=Q\left(\mathbf{t}_{2}\right)$, $\ldots, Q_{n}=Q\left(\mathbf{t}_{n}\right)$. An iteration of the EI algorithm provides the location of the next evaluation.

First, an interpolation $\hat{Q}$ of $Q$ is computed by kriging from the set of past evaluations, along with the variance of the kriging error $\hat{\sigma}^{2}(\mathbf{t})$. Denote the current minimal value by $Q_{\min }=$ $\min _{k=1, \ldots, n} Q_{k}$. Define the improvement over $Q_{\min }$ at a point $\mathbf{t} \in \mathbb{T}$ by

$$
I(\mathbf{t})=\max \left(0, Q_{\min }-Q(\mathbf{t})\right) .
$$

However, $Q(\mathbf{t})$ is unknown except at the $n$ evaluation points. Since $Q(\mathbf{t})$ is modeled by the Gaussian random process $\xi$, a natural idea is to express the expected value of $I(\mathbf{t})$, which is called the expected improvement and appears to have a very convenient analytical form:

$$
E I(\mathbf{t}) \equiv \mathbb{E}[I(\mathbf{t})]=\hat{\sigma}(\mathbf{t})[u \Phi(u)+\varphi(u)],
$$

where $\Phi(\cdot)$ is the normal cumulative distribution function, $\varphi(\cdot)$ is the normal density function, and $u$ is defined by

$$
u=\frac{Q_{\min }-\hat{Q}(\mathbf{t})}{\hat{\sigma}(\mathbf{t})} .
$$

The next evaluation point is chosen according to the highest value of the expected improvement. Since (14) is straightforward to compute, the maximization of the EI over $\mathbb{T}$ is not a problem in practice. A natural stopping criterion is when the EI is smaller than a small positive number.

Note that the EI algorithm is known to be consistent, i.e. the algorithm converges to the location of the global minimizer (under some assumptions) [Locatelli, 1997, Vazquez and Bect, 2007]. In theory, the convergence rate is unknown but it appears in practice that this type of algorithm converges very rapidly, as illustrated in our numerical studies (see next section).

\section{NUMERICAL EXAMPLES}

In this section a couple of numerical examples are selected to illustrate the presented inversion method. The parameters of the ECT configuration are shown in Table 2 (see also Fig. 1). The surface scan consists of 451 measured impedance values observed at the nodes of a rectangular grid characterized by the parameters given in Table 2. The center of the surface scan is at the origin of the $x-y$ coordinate system and the edges of the grid are parallel to the related coordinate axes.

Since no experimental measurements are available at that time, the so-called "measured impedance variation" $\left(\Delta \mathcal{Z}_{k}, k=\right.$ $1, \ldots, N$ in (4)) of the defects in the assumed test cases are obtained by numerical simulation.

The necessary initialization of our iterative process implies the evaluation of the similarity function at some well-chosen points of the parameters space. There is no "best" way to choose such initial points without complementary a priori informations, 
Table 3. Defect parameters, in case of Initialization " $A$ ". (exact/reconstructed)

\begin{tabular}{|c|c|c|c|c|c|c|c|c|}
\hline No. & $\frac{A}{m m}$ & $\frac{B}{m m}$ & $\frac{L_{1}}{m m}$ & $\frac{L_{2}}{m m}$ & $\frac{D_{1}}{\sigma}$ & $\frac{D_{2}}{\%}$ & It. & $\frac{Q_{\min }}{10^{-3}}$ \\
\hline \hline$\# 1$ & $0.50 / \mathbf{0 . 5 0}$ & $3 / \mathbf{3}$ & $3 / \mathbf{3}$ & $5 / \mathbf{5}$ & $40 / \mathbf{4 0}$ & $60 / \mathbf{6 0}$ & 57 & 0 \\
\hline$\# 2$ & $0.75 / \mathbf{0 . 7 5}$ & $1 / \mathbf{1}$ & $4 / \mathbf{4}$ & $4 / \mathbf{3}$ & $30 / \mathbf{4 0}$ & $50 / \mathbf{5 0}$ & 112 & 21.6 \\
\hline$\# 3$ & $0.75 / \mathbf{0 . 7 5}$ & $2 / \mathbf{3}$ & $7 / \mathbf{5}$ & $3 / \mathbf{3}$ & $50 / \mathbf{5 0}$ & $30 / \mathbf{5 0}$ & 95 & 28.6 \\
\hline$\# 4$ & $1.00 / \mathbf{0 . 7 5}$ & $0 / \mathbf{0}$ & $6 / \mathbf{7}$ & $6 / \mathbf{3}$ & $60 / \mathbf{6 0}$ & $40 / \mathbf{4 0}$ & 60 & 8.56 \\
\hline
\end{tabular}

Table 4. Defect parameters, in case of Initialization " $B$ ". (exact/reconstructed)

\begin{tabular}{|c|c|c|c|c|c|c|c|c|}
\hline No. & $\frac{A}{m m}$ & $\frac{B}{m m}$ & $\frac{L_{1}}{m m}$ & $\frac{L_{2}}{m m}$ & $\frac{D_{1}}{\%}$ & $\frac{D_{2}}{\%}$ & It. & $\frac{Q_{\min }}{10^{-3}}$ \\
\hline \hline$\# 1$ & $0.50 / \mathbf{0 . 5 0}$ & $3 / \mathbf{3}$ & $3 / \mathbf{3}$ & $5 / \mathbf{5}$ & $40 / \mathbf{4 0}$ & $60 / \mathbf{6 0}$ & 81 & 0 \\
\hline$\# 2$ & $0.75 / \mathbf{0 . 7 5}$ & $1 / \mathbf{1}$ & $4 / \mathbf{2}$ & $4 / \mathbf{4}$ & $30 / \mathbf{4 0}$ & $50 / \mathbf{5 0}$ & 118 & 6.98 \\
\hline$\# 3$ & $0.75 / \mathbf{0 . 7 5}$ & $2 / \mathbf{2}$ & $7 / \mathbf{7}$ & $3 / \mathbf{3}$ & $50 / \mathbf{5 0}$ & $30 / \mathbf{3 0}$ & 79 & 0 \\
\hline$\# 4$ & $1.00 / \mathbf{0 . 7 5}$ & $0 / \mathbf{0}$ & $6 / \mathbf{6}$ & $6 / \mathbf{5}$ & $60 / \mathbf{6 0}$ & $40 / \mathbf{4 0}$ & 109 & 4.73 \\
\hline
\end{tabular}

therefore it seems reasonable to spread them more or less uniformly on the parameter space. Too few points can give misleading information on the similarity function whereas too many points may be unnecessary (thus "uneconomical" in the sense of computation time). Two types of initialization are presented in the paper:

- Initialization " $A$ ": all the vertices of the $6 \mathrm{D}$ parameter hypercube $\left(2^{6}=64\right.$ points $)$ and the midpoint of the hypercube (1 point): $\Sigma 65$ points;

- Initialization " $B$ ": the same points as in Init. " $A$ ", and all the midpoints of the sides of the hypercube $(2 \cdot 6=$ 12 points): $\Sigma 77$ points.

The parameters of the cracks to be retrieved and the obtained results are reported in Table 3 and Table 4 . The depths $\left(D_{1}, D_{2}\right)$ are given in percents of the plate thickness $d$. After the initialization (65 or 77 similarity function evaluations) a fixed number of iteration cycles were performed (120 in all the cases). In the column "It." the number of cycle, in which the final best function value $\left(Q_{\min }\right)$ was found, is presented; that minimum value (related to the obtained solution) is also reported in the tables.

Table 3 and Table 4 illustrate the efficiency of EI algorithm with which an "acceptable" solution - in the sense of a low similarity function - is obtained in a few number of iterations. The performance of the algorithm is illustrated in Fig. 3. On the bottom diagrams in Fig. 3(a) and Fig. 3(b), the $\log _{10}$ of the maximal expected improvement (i.e. over the total discrete parameter space) at the related iteration cycle is presented. One can see that it is decreasing as the iterations are being performed. However, the decrease is not monotonous. As it was mentioned, in our experiments a fixed number of iterations were performed. However, it would be better to construct a stopping criterion which stops the iterations automatically right after finding the solution - to reduce the number of "unnecessary" function calls at the end. By now, no really good stopping rule has been developed. One can rely on the maximal EI value, stating that if it is lower than a small limit, the iterations can be stopped. But the choice of this limit is not obvious - as it is illustrated in Fig. 3. In Fig. 3(b), one can see that a small $Q$ value (quite close to the "final" solution in iteration No. 109) was obtained in iteration No. 60. The parameter vector related to the observation in iteration No. 60 is $\mathbf{t}=[1.0,0,6,4,60,40]$ (in the same units as in Table 4). This parameter point is close to the real solution and to the reconstructed one (see Table 4)

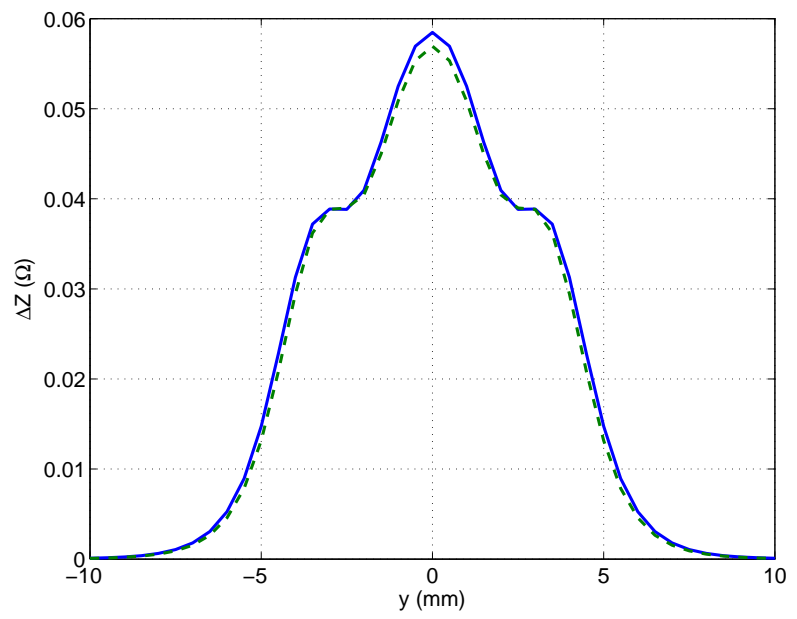

Fig. 2. Magnitude of the measured impedances in case No. \#4. Line scan along the $x=0$ line. Solid line: real crack configuration, dashed line: retrieved configuration (case Initialization " $B$ "). The measured data is almost the same in both cases, thus it is extremely difficult to make difference between the configurations.

as well. However, the maximal EI value in iteration No. 60 is almost 100 times higher than the one in iteration No. 109!

Note that the two presented performances are related to the same physical problem, only the initialization of the inversion procedure differs. In spite of this, significant differences can be found between the behaviour of the algorithm in the two cases. Thus, one can see that the choice of initial points strongly influences the latter performance. As it was mentioned above, there is no general best choice of initial points, moreover, it may also depend on the problem to be solved.

It is also important to note that the similarity function must be complicated. This is illustrated in Fig. 5: the function $Q(\mathbf{t})$ is probably quite flat around its global minimizer, or physically, different configurations can cause very similar measurable output signal.

The computations were performed on a PC with $16 \mathrm{~Gb}$ RAM and a 64 bits CPU at $3 \mathrm{GHz}$. The computation of the Green's function and of the incident field was made in advance, separately. One average iteration cycle of the optimization loop took approximately $106 \mathrm{sec}$. Almost all of that time is needed to perform the kriging prediction and EI computation, the computation of the objective function takes just a few seconds. (At first sight, one can think that the applied inversion method is more expensive-to-evaluate than the objective function itself - but note that by using this inversion method, at most some hundred function calls are needed, whereas that the parameter space $\mathbb{T}$ consists of more than 60 thousand points!)

\section{CONCLUSION}

A method for ECT inversion was presented. The stochasticbased optimization algorithm was coupled with a surface integral-based forward solver - the developed inversion method seems to be efficient in the light of the presented test cases.

The kriging interpolation of the similarity function provides a cheap surrogate model of the electromagnetic "black-box". Since this "black-box" includes electromagnetic field computation tasks, it is usually expensive-to-evaluate. This is why the 

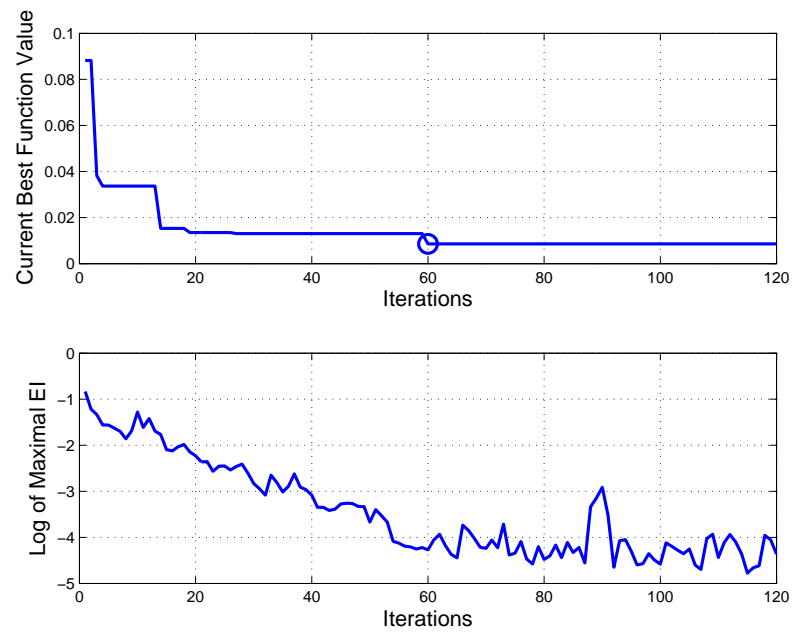

(a) Initialization " $A$ "
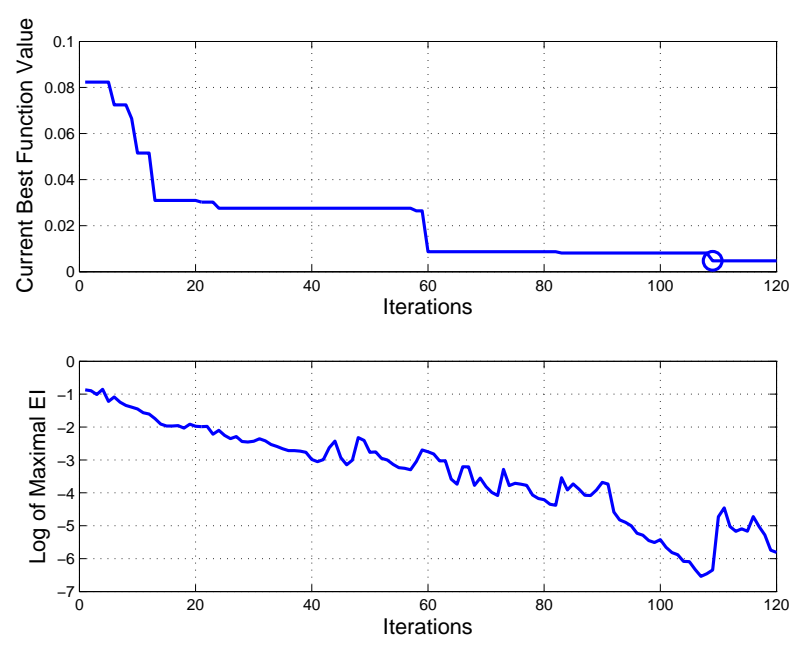

(b) Initialization " $B$ "

Fig. 3. Typical performance of the algorithm. The curves are realted to Case No. \#4, (see Table 3 and Table 4). Top: current best objective function value (the minimum of all the past observations). The found minimum is marked by a circle. Bottom: $\log _{10}$ of the maximal EI in the current cycle.

use of such surrogate models seems to be fruitful. This kind of surrogate modeling of electromagnetic phenomena has been a popular method for years and now seems to be having its "second honeymoon" [Sykulski, 2007]. Several methods exist for the solution of electromagnetic optimization problems. The main novelty of this paper is the application of such an existing (and in other domains widely used) optimization method for the inverse problem of double-crack characterization in ECT.

A couple of numerical examples illustrate the performance of the developed inversion method. The most important conclusion to be drawn is that quite few number of similarity function evaluations were enough to find an acceptable solution - even if the similarity function is "badly behaved" from the point of view of optimization. It is also important to emphasize that the performance of the algorithm is strongly influenced by the choice of initial observation points - as it was pointed out via our numerical examples.
A pitfall of the EI algorithm was also highlighted. The choice of an adequate stopping criterion is not straightforward and no general solution has been found yet. Further investigations are needed concerning this problem.

In this paper, only the brief introduction to the stochastic tools and the first results were presented - the research is far from being finished. In the authors' opinion, the use of surrogate models in ECT inversion is a hopeful idea.

\section{REFERENCES}

S. Bilicz, E. Vazquez, M. Lambert, Sz. Gyimóthy, and J. Pávó. Characterization of a 3D defect using the Expected Improvement algorithm. In Proceedings of the 13th International IGTE Symposium on Numerical Field Calculation in Electrical Engineering, Graz, Austria, September 21-24 2008. Accepted.

J. R. Bowler. Eddy-current interaction with an ideal crack. I. The forward problem. Journal of Applied Physics, 75(12): 8128-8137, June 1994.

J.P. Chiles and P. Delfiner. Geostatistics, Modeling Spatial Uncertainty. Wiley, 1999.

D.R. Jones. A taxonomy of global optimization methods based on response surfaces. Journal of Global Optimization, 21: 345-383, 2001.

M. Locatelli. Bayesian algorithms for one dimensional global optimization. Journal of Global Optimization, 10:57-76, 1997.

J. Pávó. Reconstruction of group of cracks in plate specimens using ECT impedance data. In Satish S. Udpa, Toshiyuki Takagi, József Pávó, and Rafaello Albanese, editors, Electromagnetic Nondestructive Evaluation (IV), volume 17 of Studies in Applied Electromagnetics and Mechanics, pages 204-211. IOS Press, Amsterdam, 2000.

J. Pávó and D. Lesselier. Calculation of eddy current probe signal with global approximation. IEEE Transaction on Magnetics, 42(4):1419-1422, April 2006.

J. Pávó and K. Miya. Reconstruction of crack shape by optimization using eddy current field measurement. IEEE Transaction on Magnetics, 30:3407-3410, September 1994.

J. Sacks, W.J. Welch, T.J. Mitchell, and H.P. Wynn. Design and analysis of computer experiments. Statist. Science, 4: 409-435, 1989.

J. K. Sykulski. New trends in optimization in electromagnetics. Przeglad Elektrotechniczny, 83(6):13-18, 2007. Invited paper from ISTET'07 Conference.

E. Vazquez and J. Bect. On the convergence of the expected improvement algorithm. arXiv:0712.3744v1, 2007.

J. Villemonteix, E. Vazquez, and E. Walter. An informational approach to the global optimization of expensive-to-evaluate functions. Journal of Global Optimization, 2008. URL http: //dx . doi .org/10.1007/s10898-008-9354-2. 